\title{
Traductrices, traducteurs : sommes-nous une frontière ? Sommes-nous une membrane?
}

\author{
Fabio REGATTIN \\ Università degli Studi di Udine \\ Italie
}

Résumé : La métaphore conceptuelle qui voit la traduction comme le « dépassement d'une frontière » est tellement répandue qu'elle n'apparaît presque plus comme telle : elle fait désormais partie du sens commun. Cet article essaie de la remettre en question en mettant à contribution quelques approches interdisciplinaires, qui tracent des parallèles - plus ou moins métaphoriques - entre la culture et la biologie. Nous allons explorer, à l'intérieur de ces différentes approches, la notion de frontière/limite, pour montrer une alternative qui nous paraît plus apte à capturer la complexité de la traduction : celle de la membrane.

\begin{abstract}
The conceptual metaphor that sees translation as "border crossing" is so widespread that nowadays it hardly appears as a metaphor: it has become a commonsensical notion. Our contribution tries to question it by exploring a number of interdisciplinary approaches, which draw parallels - more or less metaphorical ones between culture and biology. We will analyze, within these different approaches, the notion of border/limit, in order to show an alternative that seems more apt to capture the complexity of translation - the notion of membrane.
\end{abstract}

Mots clés : traduction, frontière, membrane, darwinisme culturel, théories systémiques

Keywords: translation, frontier, border, membrane, cultural Darwinism, systems theories

\section{En guise de préambule : de la métaphore, et de son utilité dans le discours scientifique}

Cet article s'attachera à fournir des raisons pour le remplacement d'une métaphore conceptuelle - la traduction comme "dépassement d'une frontière » - avec une autre métaphore conceptuelle - la traduction comme "passage par une membrane ». Cet objectif peut paraître à la fois ambitieux et peu important pour la traductologie. Ambitieux, parce que la métaphore de départ semble bien trop répandue pour qu'une substitution puisse avoir lieu dans le discours courant sur le domaine qui nous intéresse ; peu important, parce que de toute façon quelques lecteurs pourront penser - ce n'est pas par une nouvelle 
métaphore que la recherche sur la traduction pourra faire de véritables pas en avant.

Regarder la traduction de façon métaphorique est toutefois, à notre sens, une phase qui peut précéder - et favoriser - la réflexion sur cette activité, comme sur tout autre sujet. Comme l'affirme Max Black, les métaphores permettent en effet de voir quelque chose dans des termes nouveaux. La métaphore "sélectionne, souligne, supprime et organise des aspects du sujet principal en impliquant, sur celui-ci, des assertions qu'on porterait normalement sur le sujet subsidiaire $»^{1}$ (Black, $\left.1962: 45\right)$. Cet effet de sélection est extrêmement important dans le domaine de la communication scientifique, puisqu'il fait en sorte que toute métaphore, si tant est que nous l'adoptons, peut déterminer notre façon d'appréhender un concept : un exemple célèbre, proposé encore par Max Black (1983), est celui qui voit une bataille comme un jeu d'échecs. Les vocables du jeu vont forcément déterminer un système d’inférences qui dominera notre description, et le choix de ce lexique nous amènera à mettre au premier plan certains aspects de la bataille, alors que d'autres passeront au deuxième rang. Ainsi, le lexique des échecs «filtre et transforme, il ne fait pas qu'une sélection, mais il fait émerger certains aspects du combat qui autrement ne seraient pas visibles $»^{2}$ (Arduini et Fabbri, 2008: 40).

Tout cela nous semble suffisant pour répondre à la deuxième classe de doutes ; pour ce qui est de la première - de l'ambition de notre propos - nous serions content si ces lignes amenaient quelques lectrices et lecteurs à réfléchir sur leur conception de la traduction ; nous n'en demandons pas plus.

\section{Frontière et mouvement en traduction}

Le concept de frontière accompagne la réflexion sur la traduction (ce mot de "réflexion » étant à comprendre dans son sens le plus large : celle, plus scientifique, qui est menée par les traductologues, tout comme celle, plus intuitive, qui se retrouve dans les propos des traducteurs et des lecteurs de traductions) depuis très longtemps. ${ }^{3}$ Et pour cause; pour

\footnotetext{
1 «Selects, emphasizes, suppresses, and organizes features of the principal subject by implying statements about it that normally apply to the subsidiary subject. »

2 « Filtra e trasforma, non seleziona soltanto ma fa emergere alcuni aspetti del combattimento che non sarebbero stati visibili in altro modo. »

3 Une recherche menée grâce au système Érudit sur deux revues de premier plan pour la traductologie (Meta et TTR) et portant uniquement sur les titres, les mots-clés ou les résumés des contributions donne les résultats suivants. Frontière : 15 résultats ; frontières : 30 résultats ; border : 3 résultats ; borders :
} 
deux raisons principales, il s'accorde très bien, en effet, à notre vision intuitive du phénomène.

En premier lieu, dans la plupart des cas (et ce, bien que des contre-exemples existent), on traduit pour ouvrir à quelqu'un un accès à des propos qui lui seraient autrement incompréhensibles. Il y a donc pour celle, celui qui est exposé au texte-source avant sa traduction, et qui ne le comprend pas, un effet de barrière inévitable. On voudrait, on ne peut pas ; quelque chose - notre ignorance de la langue-source - nous empêche de comprendre ce qui se passe dans ce même idiome. Deuxièmement, l'état-nation qui est la forme politique prototypique aujourd'hui se construit, dans bien des cas, autour du couple ${ }^{1}$ " un territoire/une langue »; on a donc tendance à superposer, plus ou moins consciemment, ces deux éléments : le côté géographique (une langue se parle dans un certain territoire) se double du côté linguistique (de même que je me déplace pour aller dans un autre pays, un texte se déplace pour venir chez moi). Voici une autre raison possible du succès intuitif de cette métaphore. ${ }^{2}$

Ces deux poncifs - la langue comme barrière à la compréhension, la superposition souvent abusive de la langue et du territoire - sont peutêtre suffisants à expliquer la prolifération de métaphores qui mettent en scène un dépassement: la frontière, certes, mais aussi la traduction comme pont.

Un livre consacré aux rapports entre traduction et métaphore, et publié il y a une dizaine d'années (St. André (ed.), 2010), en répertorie plusieurs. Nous en reprendrons rapidement ici quelques-unes, dans la langue - l'anglais - où elles ont été énoncées :

[1] Building a bridge between two cultures (St. André, 2010: 1) ;

[2] Engaging in slave labour on another man's land (ibid.) ;

[3] Walking along the bank of a large river to locate a shallow place to ford it (St. André, 2010: 4) ;

[4] To translate is to move along a path to a destination. Reaching this destination (the goal of the translation) is the most important thing for the translator (Martín de León, 2010: 95) ;

[5] Jumping across an intermediate gap (Guldin, 2010: 162) ;

[6] Translations work simultaneously for two governments (Guldin, 2010: 175) ;

[7] A path, a voyage (Monti, 2010: 201) ;

17 résultats. La présence du concept dans la réflexion « naïve » sur l'activité qui nous intéresse est plus difficile à quantifier.

${ }_{1}^{1}$ Phantasmatique dans l'écrasante majorité des cas, certes ; mais nous sommes davantage intéressé ici aux constructions sociales de l'identité nationale qu'à sa réalité.

${ }^{2}$ Qui pourrait alors avoir subi une accélération depuis deux siècles environ ; il serait intéressant d'en étudier la diffusion en diachronie. 
[8] The translator is a ferryman (Roesler, 2010: 225);

[9] Translation as smuggling (Tyulenev, 2010: 241).

[10] Translation as hijacking (Tyulenev, 2010: 250).

La traduction est vue, dans l'ordre, comme un pont ou comme un bac (exemples [1] et [8]) ; comme un travail au statut ambigu (on fournit ses services à deux gouvernements à la fois, on est esclaves en territoire étranger - exemples [2] et [6]) ; comme un mouvement enfin, où le dépassement d'une frontière peut être plus (exemples [3], [5], [9] et [10]) ou moins présent (exemples [4] et [7]).

Toutes ces métaphores, certes suggestives, font globalement l'impasse sur un aspect primordial de tout acte traductif - un aspect sans lequel, nous aurions tendance à dire, il n'y a tout simplement pas de traduction. C'est la transformation, qui doit obligatoirement faire partie du processus qui nous intéresse. Ce qui passe le pont ou la frontière, ce qui est transporté d'un endroit à l'autre, la personne qui se déplace - qui est déplacée - pour aller travailler de façon volontaire ou forcée ne sont pas les mêmes objets, les mêmes personnes, qui existaient au départ.

Deux conceptions permettent de visualiser ce changement, à partir de deux perspectives différentes mais complémentaires, dans les deux cas inspirées par la biologie. La première perspective découle directement de l'évolutionnisme darwinien appliqué à la culture, et a affaire aux rapports entre objets culturels en compétition pour la survie ; la deuxième est une approche qui complémente celle-ci, et qui se tourne plutôt vers le développement interne de ces mêmes objets culturels. Dans la suite de notre article, nous allons les décrire et en tirer quelques conséquences pour la métaphore de la frontière en traduction.

\section{Darwinisme culturel et traduction : une synthèse et quelques conséquences}

Toutes les différentes approches qui se réclament, au sens large, de l'évolutionnisme culturel (à partir de Dawkins, 1976 et en passant par Sperber, 1996, Blackmore, 2000, Laland et Brown, 2011, Mesoudi, 2016 entre autres) partagent une idée de fond : le mécanisme de l'évolution darwinienne ne s'applique pas qu'aux êtres vivants, mais aussi aux objets culturels - textes, outils, images, et ainsi de suite. Ce mécanisme prévoit quatre phases, qu'on pourra rapidement résumer comme suit :

- multiplication - une entité peut donner lieu à deux ou plusieurs autres entités ;

- variation - toutes les entités ainsi produites ne sont pas identiques entre elles ;

- hérédité - les différences entre les entités peuvent être transmises d'un exemplaire à l'autre ; 
- compétition, ou sélection - les ressources disponibles dans l'environnement n'étant pas suffisantes pour chaque exemplaire, certains seulement survivront et/ou auront accès à la reproduction ; leur variabilité pourra influencer, en positif ou en négatif, leur survie.

Alors que pour le domaine biologique cet acquis n'est pas en discussion, son application à la culture est peut-être moins évidente. Pourtant, la multiplication est présente à chaque fois qu'une idée est transmise, et, pour s'en rendre compte, il suffira de penser récursivement à « l'idée d'évolution darwinienne des idées »; en effet notre point de vue, notre idée, a désormais produit des descendants chez tout lecteur attentif. La variation est également là, puisque toute idée partagée au sein d'un groupe n'a pas exactement la même forme chez tout un chacun (elle pourra par exemple être plus ou moins exacte : nous partageons avec les astronomes les idées selon lesquelles la terre n'est pas plate et tourne autour du soleil, mais chez les spécialistes celles-ci seront évidemment beaucoup plus développées) ; par ailleurs, dans toute culture tant soit peu développée, des idées différentes, voire opposées, existent et se partagent la place disponible dans nos cerveaux (les «terre-platistes ", les " géocentristes » sont hélas parmi nous !). Que dire de l'hérédité ? Pour le lecteur, la lectrice qui ne l'aurait pas déjà rencontrée, l'idée d' « évolution darwinienne de la culture » constitue encore une fois un bon exemple, puisqu'elle dépend directement de ce qu'il/elle lira dans ces pages ; un rapport de ressemblance va forcément exister entre ce texte et le concept que les lecteurs vont se former du sujet. Enfin, y a-t-il compétition? La réponse devra être, pour la quatrième fois, positive : personne en effet n'est en mesure de se rappeler tout ce qui lui arrive, tout mot qu'il/elle aurait entendu. Notre mémoire étant une ressource finie, nous l'utilisons pour retenir certaines informations au détriment d'autres informations. De plus, dans tout acte de communication, même le plus anodin, nous décidons - de façon plus ou moins consciente - de partager certaines idées au détriment d'autres idées qui, elles aussi, nous " habitent ». Or, si nous avons retenu ou exprimé une idée, c'est que certaines de ses caractéristiques la rendaient préférable en situation (à savoir, dans son environnement) par rapport à d'autres idées concurrentes. Les quatre caractéristiques étant remplies, on pourra donc considérer que les idées peuvent, elles aussi, être soumises à la sélection naturelle.

Que dire de la traduction, dans tout cela ? Les rapports entre cette activité et l'évolution culturelle ont été maintes fois pris en compte par la traductologie (Vermeer, 1997, Hu, 2003, Chesterman, 2016, par exemple), avec des résultats variables. Nous avons proposé récemment (Regattin, 2018 et 2019) que traduction et évolution culturelle seraient en quelque sorte coextensives. En effet, les quatre conditions nécessaires à l'évolution sont nécessaires pour la traduction aussi : multiplication (le 
texte en langue-cible s'ajoute généralement au texte en langue-source) ; variation (ce n'est forcément pas le même texte : cela vaut pour la traduction interlinguistique tout comme pour toute autre forme de traduction - intralinguistique ou intersémiotique (Jakobson, 1959); hérédité (la traduction est déterminée par son texte-source - dans le cas contraire, on se trouverait dans la création autonome e non pas dans la traduction) ; enfin, sélection (tout texte n'est pas traduit, et même dans les textes traduits, on choisira une dominante à respecter, au détriment des autres aspects du texte-source; encore une fois, certaines idées seulement seront sélectionnées et non pas d'autres).

Dans cette vision de la traduction, on peut considérer que la pratique qui nous intéresse est tout de même fonctionnelle au dépassement d'une frontière, qui peut bien être considérée comme telle. En effet, la séparation entre les langues semble se comporter - pour les objets culturels exprimés par la voie linguistique - de façon semblable à n'importe quelle barrière physique suffisamment importante (par exemple un océan, une chaîne de montagnes) pour les êtres vivants. Une " frontière », dans ce cas, existe - celle qui sépare les langues ou du moins celle qui s'oppose à l'intercompréhension $;^{1}$ parler de transportdéplacement est toutefois erroné, et il faudrait plutôt choisir des termes tels que filiation-évolution-adaptation. Un premier résultat de cette lecture est en effet le suivant : en traduction, rien ne se déplace. L'objet qui se trouvera de l'autre côté de la frontière est en effet un descendant modifié de l'original, conçu dès le départ de cet autre côté. Linguistiquement, il reste envisageable de parler de dépassement d'une frontière, mais seulement dans des cas-limite : les emprunts à une langue étrangère ou des citations directes (par exemple, des chansons) qui passeraient inchangés d'une aire linguistique à une autre. Dans ce cas, la frontière est effectivement passée, mais il n'y a pas de traduction !

Notre première proposition sera donc la suivante: la seule " frontière » qui existe en traduction est incarnée par la traductrice ou le traducteur. C'est elle, c'est lui qui - faisant partie des deux territoires à la fois -, tout en maintenant le texte source dans son propre environnement, crée un nouveau texte qui évoluera dans le deuxième système linguistique. Peut-on vraiment, dès lors, parler de frontière ? La

\footnotetext{
${ }^{1}$ Nous ne faisons pas référence, ici, à la diffusion géographique des langues ou, plus bas, des textes. Des limites géographiques sont évidemment dépassées à chaque fois qu'un locuteur d'une certaine langue, ou un livre par exemple, se déplace au-delà d'une frontière (à chaque fois que je sors des frontières italiennes ou que je ramène un livre, un journal de mon voyage, par exemple). La perspective que nous adoptons ici est celle du «monde 3 » dont parle Karl Popper (1972), le «monde des idées », relativement autonome du monde physique. Dans celui-ci, on peut considérer qu'il existe frontière entre deux systèmes sémiotiques dès qu'il n’y a pas intercompréhension.
} 
traduction semble avoir un comportement paradoxal : elle se situe à une frontière ; toutefois, elle ne la dépasse jamais.

\section{Théories (bio)systémiques et traduction : quels enseignements?}

Comme nous nous trouvons dans le champ des analogies, peutêtre y-a-t-il lieu de remplacer l'image de la frontière avec une image différente, qui pourrait produire des inférences plus proches de la réalité des faits - d'ailleurs, le pouvoir des analogies pour la formation et l'appréhension de la réalité et du discours scientifique est bien connu (Black, 1979, Itkonen, 2005). Des idées pour aller chercher cette autre image pourraient nous venir du domaine des théories systémiques, et mieux encore (pour garder l'isotopie biologique, qui semble prometteuse) de ce sous-secteur des études culturelles qui s'inspire des théories systémiques en biologie. ${ }^{1}$

\subsection{Youri Lotman et sa sémiosphère}

Une référence incontournable est, dans ce domaine, Youri Lotman, avec le concept de sémiosphère $\left(1999^{2}\right)$, conçu - par analogie à partir de l'idée de biosphère développée dans les années 1920 par Wladimir Vernadsky. La sémiosphère serait, pour Lotman, l'espace à l'intérieur duquel la culture peut vivre et se développer, où la sémiose est possible. Tout processus sémiotique aurait lieu à l’intérieur de cet espace.

\footnotetext{
${ }^{1}$ Deux remarques préalables : nous ne ferons qu'effleurer des réflexions qui sont, bien évidemment, beaucoup plus complexes, en nous intéressant uniquement à l'aspect qui nous retient ici, à savoir les notions de frontière et/ou de membrane. Cet aspect de sélection est, par ailleurs, double : il y aura, en premier lieu, la simplification que notre lecture entraînera nécessairement ; et nous laisserons de côté, de la même manière, plusieurs théories qui pourraient être mises à contribution mais qui, pour nos propos, ne feraient que reproduire des idées très semblables. Nous pensons par exemple à la théorie des systèmes sociaux de Niklas Luhmann (Tyulenev, 2012) ou même, pour certains de ses aspects, à la théorie du polysystème d'Itamar Even-Zohar (1990).

${ }_{2}$ N'ayant pas pu accéder à l'intégralité de la traduction française, nous nous sommes souvent appuyé sur la version italienne du texte (Lotman, 1985) ; c'est pourquoi nous allons essayer de limiter au minimum les citations littérales. Là où celles-ci seraient indispensables, nous en fournirons une version de service en français dans le texte, tout en espérant que ce double passage n'aura pas trop déformé la pensée de Lotman, et nous donnerons le texte italien en note. Pour cette même raison, considérant les règles de translittération du cyrillique en français et en italien, le prénom de Lotman va changer, en bibliographie, selon les textes cités.
} 
Cet univers sémiotique peut, bien sûr, être considéré comme un ensemble de textes et de langages séparés les uns des autres; la perspective de Lotman $(1985: 58)$ vise toutefois à remplacer en quelque sorte l'étude des briques par l'étude de la construction, à donner priorité à l'ensemble du système. Lotman considère tout de même que la perspective systémique peut être adoptée pour la sémiosphère dans son ensemble aussi bien que pour des sémiosphères dont celle-ci serait à son tour composée. Chaque partie serait ainsi, en même temps, un entier doté d'une indépendance structurelle, et isomorphe à l'ensemble (1985, 65). La sémiosphère peut donc être conceptualisée comme un système de systèmes, où un rôle primordial est joué par la notion de frontière. ${ }^{1}$ Celleci marque la limite entre l'intérieur et l'extérieur de chaque sémiosphère ; c'est « l'ensemble des points qui appartiennent en même temps à l'espace intérieur et à l'espace extérieur, [...] la somme des 'filtres' linguistiques de traduction ".$^{2}$ La sémiosphère est un espace fermé : elle ne peut avoir de rapport ni avec des non-textes (pour la sémiosphère en général), ni avec des textes qui lui sont étrangers d'un point de vue sémiotique (pour les sémiosphères enchâssées).

Pour que ces faits acquièrent une réalité pour la sémiosphère, ils devront être sémiotisés ou traduits dans un langage présent à son intérieur : "La fonction de toute frontière, de toute pellicule (depuis la membrane de la cellule vivante jusqu'à la biosphère [...]) est de contrôler, de filtrer et d'adapter ce qui est externe à ce qui est interne $», 3$ affirme à ce propos Lotman (1999: 35). Ces deux actes se résument en un seul : la traduction. Celle-ci est par définition annexionniste, puisque les mécanismes de traduction appartiennent nécessairement à la structure de la sémiosphère (1985: 61). Puisque la frontière est un élément nécessaire pour la sémiosphère, celle-ci aura toujours besoin d'un environnement extérieur non organisé; lorsqu'un tel environnement n'existe pas, la sémiosphère le créera. « La culture, en effet, ne crée pas

\footnotetext{
${ }^{1}$ Nous reviendrons plus bas sur le choix de ce mot, qui est justement celui dont nous voudrions nous débarrasser.

2 « L'insieme di punti che appartengono allo stesso tempo allo spazio interno e a quello esterno, [...] la somma dei 'filtri' linguistici di traduzione » (Lotman, 1985: 58).

3 Lotman fait ici un pas dans celle qui nous semble être la bonne direction, en assimilant la frontière et la membrane - il semble considérer par ailleurs que celle-ci en est l'exemple le plus simple. Il y a toutefois, nous semble-t-il, une différence entre les deux termes, qui mérite qu'on s'y arrête. En effet, si toute frontière sépare, elle ne transforme pas forcément au passage ce qui lui était externe. D'où l'utilité de remplacer carrément ce mot par celui de membrane. Nous y reviendrons.
} 
que son organisation interne; elle crée aussi un type à elle de désorganisation externe $»^{1}$ (Lotman, $\left.1985: 62\right)$.

\subsection{La réflexion (bio)sémiotique de Kobus Marais}

Récemment, le chercheur sud-africain Kobus Marais (2019) a développé un cadre théorique qui rappelle sous plusieurs points de vue celui qui précède. Cela n’a d'ailleurs rien d'étonnant, Marais se réclamant d'une discipline (la biosémiotique) qui descend en ligne droite de la réflexion de Lotman. L'ouvrage de Marais est une tentative de rendre compte de tout phénomène portant les préfixes trans- ou inter- (2019: 6) : un élargissement sensible, donc, du champ de la traduction qui comporte le passage " des objets à l'inter- entre les objets ».2 Au centre de tous les processus traductifs est placé le concept de sémiose, qui semble parfois se confondre avec celui de traduction. Celle-ci devient, dans cette lecture élargie, un élément central non seulement pour la communication, mais pour la vie au sens large. La sémiose est traduction ( «Il n'y a qu'une manière de créer de la signification, et c'est en traduisant des signes en d'autres signes »3), et elle est consubstantielle à la vie même, puisque "tous les êtres vivants prennent part dans les processus sémiotiques, ce qui signifie que ceux-ci sont un élément constitutif de la vie ».4 Dans ce cadre, Marais revient sur le concept de frontière, mais pour le remettre en question et proposer à son tour la métaphore de la membrane. Celle-ci crée à la fois une séparation et une connexion entre le dedans et le dehors du système : "L'interaction à travers une membrane est l'activité sémiotique la plus simple qui soit ».5

Dans ces visions « de l'intérieur », où l'objet étudié n'est plus le texte mais la culture dans laquelle il évoluera, on retrouve une idée-clé : des choses « rentrent » dans un nouveau système, certes, mais le système les transforme. C'est que chaque système (vivant ou pas, cela n'a pas d’importance) vit, évolue, dans un environnement différent, puisqu'il sélectionne et pertinentise des aspects différents de l'environnement commun - ceux qui sont utiles à sa propre survie ; il serait possible de

\footnotetext{
1 « La cultura non crea infatti soltanto la sua organizzazione interna, ma anche un proprio tipo di disorganizzazione esterna. »

2 « From things to the inter- between things » (Marais, 2019: 129).

3 « Meaning is created in one way only, and that is by translating signs into signs » (Marais, 2019: 122).

4 « All living organisms participate in semiotic processes which means that sign processes are constitutive for life » (Marais, 2019: 115).

5 "Interaction through a membrane is the most basic semiotic activity » (Marais, 2019: 153).
} 
mettre à contribution ici la notion d'Umwelt ${ }^{1}$ telle qu'elle a été développée par Jakob von Uexküll (Chamois, 2016).

\section{Bilan}

Il est possible, à partir de cette double lecture, de considérer que les métaphores biologiques de la culture permettent d' " améliorer », pour ainsi dire, une lecture qui revient constamment dans le discours sur la traduction. On pourra poser alors qu'une frontière sépare bien - et unit tous ces objets qui ne changent pas d'un côté et de l'autre : les êtres vivants, par exemple, mais aussi cette sous-catégorie de produits culturels qui ne font pas l'objet de modifications (comme nous l'avons dit plus haut: chansons, emprunts, ${ }^{2}$ " mèmes Internet », et ainsi de suite). Par contre, une membrane sépare, unit et modifie ce qui se trouve à l'intérieur ou à l'extérieur d'un système donné. Ce « modifie » fait toute la différence entre les deux métaphores. Dans le premier cas, l'objet culturel restera un corps étranger, qui ne sera pas forcément compris à l'intérieur du nouveau système dans lequel il devra évoluer; ce n'est qu'avec la traduction, avec la modification qu'elle entraîne, que celui-ci pourra être intégré et pertinentisé à l'intérieur de son nouvel espace de diffusion. ${ }^{3}$ Remplacer de façon systématique la métaphore de la frontière avec celle de la membrane permettrait de faire émerger cette différence, et de faire comprendre que presque rien (et rien du tout, en traduction) ne rentre inchangé dans un nouveau système linguistique.

\footnotetext{
${ }^{1}$ En gros, et de façon très simplifiée : quand je promène mon chien dans un parc, nous évoluons dans un environnement qui est, en même temps, semblable et différent. Semblable, parce que c'est, objectivement, le même environnement ; différent, parce que chacun de nous va se focaliser, à l'intérieur de cet environnement, sur des réalités et des signaux différents. Son environnement sera surtout olfactif, et très riche de ce point de vue-là ; le mien sera plutôt visuel. Un arbre sera pour moi quelque chose de beau - je pourrai même deviner un visage sur son écorce - ou une source d'ombre, de fraîcheur ; pour lui, si j'en juge naïvement à son comportement, ce sera surtout un lieu privilégié de « communication urinaire » avec ses pairs...

2 ...et encore! Malgré l'identité formelle, l'on sait très bien qu'un emprunt ne garde pas toujours (pas souvent) la même signification dans son nouvel environnement - pensons au mot anglais people et à son usage sous forme adjectivale en français.

3 Une formule à retenir : Wuthering Heights dépasse les frontières quand elle est chantée par Kate Bush, mais pas quand elle est écrite par Emily Brontë.
} 


\section{Références bibliographiques}

ARDUINI, Stefano, et Roberta FABBRI. Che cos'è la linguistica cognitiva? Roma, Carocci, 2008.

BLACK, Max. Models and Metaphors. Ithaca, Cornell University Press, 1962.

BLACK, Max. "More about metaphor ». In : Andrew Ortony (ed.), Metaphor and Thought. Cambridge, Cambridge University Press, 1979: 19-43.

BLACK, Max. Modelli, archetipi, metafore. Parma, Pratiche, 1983.

BLACKMORE, Susan. The Meme Machine. Oxford, Oxford University Press, 2000.

CHAMOIS, Camille. "Les enjeux épistémologiques de la notion d'Umwelt chez Jakob von Uexküll ». In : Tétralogiques 21, $2016:$ 171-194.

CHESTERMAN, Andrew. Memes of Translation. The Spread of Ideas in Translation Theory, $2^{\text {nd }}$ edition. Amsterdam-Philadelphia, John Benjamins, 2016.

DAWKINS, Richard. The Selfish Gene. Oxford, Oxford University Press, 1976.

EVEN-ZOHAR, Itamar. Polysystem Studies. Poetics Today 11(1), 1990.

GULDIN, Rainer. "Metaphor as a metaphor for translation ». In : James St. André (ed.). 2010 : 161-191.

HU, Gengshen. "Translation as adaptation and selection". Perspectives Studies in Translatology 11(4), $2003: 283-291$.

ITKONEN, Esa. Analogy as Structure and Process. Amsterdam-Philadelphia, John Benjamins, 2005.

JAKOBSON, Roman. " On linguistic aspects of translation ». In : Reuben Arthur Brower (ed.). On Translation. Cambridge: Harvard University Press, 1959: 232-239.

LALAND, Kevin N., et Gillian R. Brown. Sense \& Nonsense. Evolutionary Perspectives on Human Behaviour, $2^{\text {nd }}$ edition. Oxford, Oxford University Press, 2011.

LOTMAN, Yurij. La semiosfera: l'asimmetria e il dialogo nelle strutture pensanti. Venezia, Marsilio, 1985.

LOTMAN, Youri. La sémiosphère. Limoges, Presses Universitaires de Limoges, 1999.

MARAIS, Kobus. A (Bio)Semiotic Theory of Translation. The Emergence of Socio-Cultural Reality. London-New York, Routledge, 2019.

MARTÍN DE LEÓN, Celia. "Metaphorical models of translation: transfer vs. imitation and action ». In : James St. André (ed.), 2010 : 75-108.

MONTI, Enrico. "Metaphors for metaphor translation ». In : James St. André (ed). $2010:$ 192-210.

MESOUDI, Alex. "Cultural evolution: a review of theory, findings and controversies ». Evolutionary Biology 43(4), 2016 : 481-497.

POPPER, Karl. La Connaissance objective. Paris, Flammarion, 1972.

REGATTIN, Fabio. Traduction et évolution culturelle. Paris, L'Harmattan, 2018.

REGATTIN, Fabio. «Biologiser les idées ? Traduction et darwinisme culturel : rapports, apports, passé, futur ». In : Syn-thèses 9-10, 2019: 56-74.

ROESLER, Stéphanie. «Yves Bonnefoy's metaphors on translation ». In : James St. André (ed.). $2010: 211-240$.

SPERBER, Dan. La Contagion des idées. Paris, Odile Jacob, 1996. 
ST. ANDRÉ, James (ed.). Thinking through Translation with Metaphors. Manchester, St. Jerome Publishing, 2010.

ST. ANDRÉ, James. "Translation and metaphor: setting the terms ». In : St. André, James (ed.). 2010: 1-16.

TYULENEV, Sergey. "Translation as smuggling ». In : James St. André (ed.). 2010: 241-274.

TYULENEV, Sergey. Applying Luhmann to Translation Studies. Translation in Society. London-New York, Routledge, 2012.

VERMEER, Hans J. « Translation and the "meme" ». In: Target 9(1), $1997: 155$ 166. 